\title{
Structure of the Caulobacter Crescentus suppressor of copper sensitivity protein C
}

\author{
Guillaume A. Petit ${ }^{1}$, Karrera Djoko², Jennifer L. Martin ${ }^{1,3}$, Maria A. Halili ${ }^{1}$ \\ ${ }^{I}$ Griffith Institute for drug discovery, Griffith University, Nathan, QLD, Australia \\ ${ }^{2}$ Department of Biosciences, Durham University, UK \\ ${ }^{3}$ Vice-Chancellor's Unit, University of Wollongong, NSW, Australia \\ guillaume.petit@griffithuni.edu.au
}

Bacterial oxidoreductase enzymes are found in the periplasm of bacteria and are involved in protein thiol oxidation, reduction and isomerisation. These proteins contribute to folding and correcting disulfide bonds in a wide range of substrates, including virulence factors. Among oxidoreductases, the disulfide bond forming protein A (DsbA) for example, has been thoroughly studied, characterised and shown to be involved in the virulence of multiple pathogenic bacteria. More recently, other oxidoreductases have received attention too. This is the case for the suppressor of copper sensitivity proteins (SCS). One member of this family, ScsC, has been found to contribute to copper resistance in Salmonella enterica serovar Typhimurium [1], and, is involved in disulfide bond isomerisation in the periplasm of the bacterium Proteus mirabilis [2]. The C-terminal catalytic domain of ScsC has an architecture similar to that of DsbA, displaying a conserved thioredoxin fold, including a CXXC catalytic motif and an embedded $\alpha$-helical domain, however the N-terminal domain, responsible for the quaternary structure of the protein, varies strongly in between the proteins from different bacteria species. In $S$. Typhimurium the protein is monomeric while in P. mirabilis, it is trimeric. More interestingly, the catalytic activity of the protein seems to depend on these C-terminal oligomerisation domains. Here we report the crystal structure of a new trimeric $\mathrm{ScsC}$ protein from the model bacterium Caulobacter crescentus, termed CcScsC. The trimerization domain of $\mathrm{CcScsC}$ is comprised of a long N-terminal $\alpha$ helix, which assemble via hydrophobic contact between the helices of the different protomers as well as a number of electrostatic interactions between their charged residues. In addition CcScsC is shown to bind copper (I) with picomolar affinity and to have isomerase activity comparable to the known bacterial isomerase E. coli DsbC. In conclusion, we report the structure of a trimeric bacterial oxidoreductase, with a role in protein thiol isomerisation and copper binding.

1. Subedi, P., Paxman, J. J., Wang, G., Ukuwela, A. A., Xiao, Z. \& Heras, B. (2019). The Journal of biological chemistry $29415876-15888$

2. Furlong, E. J., Choudhury, H. G., Kurth, F., Duff, A. P., Whitten, A. E. \& Martin, J. L. (2018). The Journal of biological chemistry 293, 5793-5805.

Keywords: Bacterial oxidoreductases, disulfide bond, copper resistance 